\title{
The Control of Surface Friction on the Scales of Baroclinic Eddies in a Homogeneous Quasigeostrophic Two-Layer Model
}

\author{
CHIUNG-YIN CHANG \\ Program in Atmospheric and Oceanic Sciences, Princeton University, Princeton, New Jersey \\ ISAAC M. HELD \\ NOAA/Geophysical Fluid Dynamics Laboratory, Princeton, New Jersey
}

(Manuscript received 1 December 2018, in final form 7 March 2019)

\begin{abstract}
In idealized models of the extratropical troposphere, both $\beta$ and surface friction can control the equilibrated scales of baroclinic eddies by stopping the inverse cascade. A scaling theory on how surface friction alone sets these scales was proposed by Held in 1999 in the case of a quadratic drag law. However, the theory breaks down when friction is modeled by linear damping, and there are other reasons to suspect that it is oversimplified. An ideal system to test the theory is the homogeneous two-layer quasigeostrophic model in the $\beta=0$ limit with quadratic damping. This study investigates some numerical simulations of the model to analyze two causes of the theory's breakdown. They are 1) the asymmetry between two layers due to confinement of friction to the lower layer and 2) deviation from a spectrally local inverse energy cascade due to the spread of wavenumbers over which energy is input into the barotropic mode. The former is studied by comparing the simulations with drag appearing asymmetrically or symmetrically between the two layers. The latter is addressed with a heuristic modification of the theory. A regime where eddies equilibrate without an inverse cascade is also examined. A comparison is then made between quadratic and linear drag simulations. The connection to a competing theory based on the dynamics of equivalent barotropic vortices with thermal signatures is further discussed. Finally, we present an example of an inhomogeneous statistically steady state to argue that the diffusivity obtained from the homogeneous model has relevance to more realistic configurations.
\end{abstract}

\section{Introduction}

Ever since Phillips (1956)'s pioneering work, the quasigeostrophic two-layer model has repeatedly proven itself to be useful for understanding the baroclinic eddy dynamics in the extratropical troposphere. To better study how eddy statistics depend on mean flows, the model can be further simplified with a fixed background zonal flow with vertical shear that is independent of latitude and that assumes the deviations from this flow to be horizontally homogeneous. This is known as the homogeneous quasigeostrophic two-layer model, and it has also provided some insights into the dependence of eddy amplitudes and eddy fluxes on the background state (Haidvogel and Held 1980; Larichev and Held 1995, hereafter LH95; Held and Larichev 1996; Thompson and Young 2006, hereafter TY06, 2007).

Corresponding author: Chiung-Yin Chang, cychang@princeton.edu
However, the flows in this idealized system are still complex enough to prevent us from fully understanding the control of eddy scales by the parameters of interest. One such parameter is the meridional gradient of Coriolis parameter $\beta$. The presence of $\beta$ introduces flow anisotropy, Rossby waves, coherent structure as zonal jets, and asymmetry between the two layers. These complexities have raised challenging questions as well as stimulated theories that attempt to address them from different perspectives. Yet the theories differ from each other, and a general consensus has not been reached (Held and Larichev 1996; Lapeyre and Held 2003; Thompson and Young 2007; Chai 2016).

To test our understanding of the homogeneous quasigeostrophic two-layer model, reconciling these theories is important. It is nonetheless also very difficult. To reduce the problem to a smaller one in which we can likely make progress, we focus on the homogeneous quasigeostrophic two-layer model in the limit of $\beta=0$. 
At the very least, eliminating $\beta$ enables us to gain a better sense of the extent of complexities that are not directly associated with $\beta$. In particular, large-scale surface friction and its roles in affecting the eddy statistics often receive less attention when $\beta$ is present. For a strongly baroclinically unstable flow, the scale of energy containing eddies is determined by the mechanism stopping the barotropic inverse energy cascade (Rhines 1977; Salmon 1978, 1980). This can either be $\beta$ or friction (or both), but it is often assumed that $\beta$ is large enough to stop the cascade, that is, channel the energy into zonal flows that do not contribute to meridional transport, before friction becomes significant (e.g., Rhines 1975). To make further progress in understanding how the cascade is terminated when both are present, we need to better understand how friction alone stops the cascade. This understanding will help us determine if this limiting case might itself have some relevance in some atmospheric (and oceanic) circulations of potential interest.

In the attempt to assess the importance of surface friction in this respect, Held (1999, hereafter H99) proposed a scaling theory to estimate the eddy scales when the cascade stopping mechanism is nonlinear (quadratic) friction. The friction in the atmospheric planetary boundary layer is typically parameterized as a quadratic drag. When imposing quadratic drag on layer models of the sort considered here, one needs to divide the nondimensional drag coefficient $C_{D}$ in the surface stress law by the depth of the lowest model layer $H$, since it is only in this combination that the surface stress is felt by the model. This results in an inverse frictional length, $L_{D}^{-1} \equiv C_{D} H^{-1}$. The strength of this type of frictional damping is then determined by $L_{D}$. If the large-scale drag is responsible for essentially all of the dissipation of kinetic energy $\varepsilon_{d}$, then we expect that $\varepsilon_{d}$ is scaled as

$$
\varepsilon_{d} \sim L_{D}^{-1} V^{3}
$$

where $V$ is a characteristic velocity scale. According to LH95, if the two-layer baroclinic turbulence is sufficiently unstable so that kinetic energy is mostly barotropic, $V$ can be thought as the typical velocity in the barotropic flow at the energy-containing eddy length scale $L$. Suppose that the barotropic energy spectrum is well approximated by Kolmogorov's inertial range theory, then $V$ can be solely determined by $L$ and the rate of inverse energy cascade $\varepsilon_{c}$ via dimensional analysis,

$$
V \sim L^{1 / 3} \varepsilon_{c}^{1 / 3} .
$$

Given $\varepsilon_{d} \approx \varepsilon_{c}$, Eqs. (1) and (2) together indicate that, if the cascade is stopped by quadratic drag alone, the energy-containing eddy length scale should be proportional to the frictional length:

$$
L \sim L_{D},
$$

which is the same as the eddy scaling for two-dimensional turbulence stirred at small scales and damped by quadratic drag (Grianik et al. 2004). Building on this scaling relation, one can further estimate an eddy diffusivity $D$ from mixing-length theory:

$$
D \sim V L .
$$

If the eddy heat fluxes are mostly due to the passive mixing of temperature (the baroclinic streamfunction in the two-layer model) by the energy-containing barotropic eddies and therefore are diffusive-like, the available potential energy production $\varepsilon_{p}$ in the homogeneous quasigeostrophic two-layer model can be expressed as

$$
\varepsilon_{p} \sim D U^{2} \lambda^{-2},
$$

where $U$ is the imposed vertical shear (proportional to temperature gradient) and $\lambda$ is the radius of deformation (prescribed in a quasigeostrophic model). One factor of $U$ in this expression arises from the temperature gradient in the expression for available potential energy generation; the other arises from expressing the eddy heat flux as a diffusivity times a temperature gradient. Provided that $\varepsilon_{p} \approx \varepsilon_{d}$, Eqs. (1), (2), (4), and (5) together also suggest that

$$
\begin{aligned}
& V \sim L_{D} U \lambda^{-1}, \\
& D \sim L_{D}^{2} U \lambda^{-1} .
\end{aligned}
$$

This theory can therefore predict how the characteristic eddy length, velocity, and diffusivity change with $L_{D}$. To see this more clearly, we can also nondimensionalize Eqs. (3), (6), and (7) with $U$ and $\lambda$. This nondimensionalization has the effect of clarifying the difference between this theory and Stone's (1972), in which $\lambda$ and $U$ are assumed to be the scales for $L$ and $V$. The $\mathrm{H} 99$ scaling predicts that $L \lambda^{-1}$ and $V U^{-1}$ are both proportional to $L_{D} \lambda^{-1}$, which is a measure of the strength of inverse cascade. A corollary of this result is that an inverse cascade is only expected when $L_{D}$ is larger than $\lambda$.

Another way of stating the above prediction is that the extent of an inverse energy cascade depends on the ratio of two dimensionless numbers: the Prandtl ratio $f / N$ and the nondimensional drag coefficient $C_{D}$. Recall $L_{D}^{-1} \equiv C_{D} H^{-1}$ and suppose that the same $H$ appears in the definition of $\lambda \equiv N H / f$ (while they do not need to be the same in general), then the ratio $L_{D} \lambda^{-1}=(f / N) C_{D}^{-1}$. 
For the typical atmospheric values, $f / N \approx 10^{-2}$. If $C_{D} \approx 10^{-3}$ such as over the oceans, this theory suggests that there should be a significant inverse energy cascade; in contrast, if $C_{D} \approx 10^{-2}$ such as over the land surface, there should be little cascade. The relevance of this ratio of the nondimensional drag coefficient and the Prandtl ratio for whether or not the eddies are strongly damped in this sense is therefore an interesting implication of this scaling argument.

There have been some numerical studies on how eddy scales depend on quadratic drag (in the absence of $\beta$ ), but none of their simulations fully support H99's eddy scaling or the suppression of inverse cascade when drag becomes sufficiently large (Arbic and Scott 2008; Jansen et al. 2015). A possible explanation for this simulation-theory inconsistency is that the theory fails to distinguish the diverse velocity scales in the twolayer system. It oversimplifies the difference between the velocity scale relevant for frictional dissipation [in Eq. (1)] and the velocity scale relevant for inverse cascade [in Eq. (2)]. Compared to the two-dimensional system, this additional level of complexity may be attributed to the layer asymmetry caused by friction only appearing in the bottom layer. If friction appears symmetrically between the two layers, eddy statistics in the two layers would be identical. This, along with the fact that most kinetic energy is barotropic when friction is weak, implies all the velocities should scale closely with each other. However, even if the eddy statistics in the two layers are indeed identical, the two-layer system still has 2 degrees of freedom in the vertical. Interactions between barotropic and baroclinic modes can still result in eddy statistics of the barotropic mode in this two-layer system differing considerably from eddy statistics of a two-dimensional system [i.e., Eq. (2) may be invalid]. If the theory fails only because of the layer asymmetry, improving the theory would only require an extra consideration on the bottom heaviness of friction. If it also has to do with the latter, we would need to reassess the assumptions in LH95 more fundamentally.

In fact, if one had considered the case of linear drag and tried to use the assumptions made in LH95 to derive a scaling theory that is analog to H99's theory for quadratic drag, one would have arrived at a conclusion that the linear drag (alone) cannot halt the inverse energy cascade and the system would not equilibrate. In such a scenario, there would be no statistically steady state unless the effect of finite domain size is considered. A related aspect of why LH95 theory with linear damping may be invalid has indeed been addressed in TY06, who studied the simulations that are conducted with the model configuration similar to
LH95 and with the bottom linear drag strong enough to prevent the eddy mixing length reaching the domain size. They have found that the model did equilibrate with well-defined eddy statistics. Also, different from what is expected by LH95, most temperature anomalies are seen to be concentrated in the cores of the coherent vortices persisting in the domain. This leads them to argue that the heat transport is due to the drift of these vortices, rather than temperatures being passively mixed by the barotropic eddies. It therefore questions the relevance of the "dual cascade" picture on which LH95 and H99 are based.

The goal of this study is to determine whether and how these different complexities lead to the breakdown of H99's theory, and we consider the homogeneous quasigeostrophic two-layer model in the limit of $\beta=0$ with quadratic drag as the ideal system to tackle the problem. To study how the layer asymmetry caused by the bottom friction affects the eddy scaling, we conduct numerical simulations with two types of frictional damping: one with quadratic drag appearing in the bottom layer only (referred to as asymmetric drag) and the other with quadratic drag appearing symmetrically between the two layers (referred to as symmetric drag). In section 2, we diagnose the eddy scales in these two sets of simulations and compare their dependence on the nondimensional damping strength to discern the differences resulting from layer asymmetry. Since the layer asymmetry is completely removed in the symmetric drag configuration, we can also test its eddy scaling with H99's theoretical prediction more cleanly to isolate the other complexities related to the uncertainties in the theory. The observed deviations of symmetric drag simulations from the prediction has motivated a heuristic theory that is modified from LH95's assumptions to take the spectral nonlocality of barotropic energy input into account. We present this modified theory in section 3 and check it with the asymmetric drag simulations in section 4 to verify whether the complexity due to the spectral nonlocality can be well separated from the complexity due to the layer asymmetry. We also investigate the strong symmetric drag regime where the eddies equilibrate without an inverse cascade. The eddy scaling and the sensitivity of equilibrated eddy statistics to initial conditions in this regime are explained (section 5).

Built on these quadratic drag results, we then discuss how the modified theory, because of the departure from H99 scaling, can make a well-defined prediction for the case with linear damping (section 6). After clarifying the relations between quadratic and linear drag, we are eventually better posited to address the question why the modified scaling, which is still based on the 
LH95's physical picture, can be useful despite its apparent conflict with TY06's findings. We discuss this issue in section 7. Finally, we show an example of a series of simulations in an inhomogeneous channel model with $\beta=0$ to illustrate that the diffusivity obtained from the homogeneous model can be used to predict the behavior of the baroclinic unstable jets in these inhomogeneous simulations (section 8). We conclude with the discussion of some implications and remaining questions on understanding the baroclinic eddy scales in more complicated and realistic flows (section 9).

\section{Asymmetric versus symmetric drag simulations}

We set up the homogeneous two-layer quasigeostrophic model in a way that closely resembles the one investigated by LH95. The model solves the same set of the equations as in LH95 [cf. their Eqs. (1)-(3)] except for the replacement of the large-scale dissipation in the linear drag form with the quadratic drag form described in Grianik et al. (2004). The model equations are presented in the appendix along with details of the numerics. As noted there, the value of $U$ is irrelevant in the case with quadratic drag since there are no time scales, only length scales, defined by the model [changing $U$ only has the effect of renormalizing velocities as shown in Arbic and Scott (2008)]. Following LH95's notations, we express the model parameters in the length unit as wavenumbers ( $k$; the first wavenumber $k=1$ is the wavenumber of the domain length): $k_{d}$ denotes the wavenumber of $\lambda$ and $c_{D}$ denotes the wavenumber of $L_{D}$. Hence, the single nondimensional parameter characterizing the system is $c_{D} k_{d}^{-1}$, whose physical meaning is essentially the nondimensional damping strength. Varying the value of $c_{D} k_{d}^{-1}$, we conduct a series of model simulations (Fig. 1). We initialize the model from infinitesimal perturbations in the streamfunction field, and we compute the eddy statistics from the time series of the statistically steady state. These eddy statistics include the characteristic eddy length scale $L$, velocity $V$, and diffusivity $D$ that we have introduced in section 1 and will now be more precisely defined in the same way as TY06:

$$
\begin{aligned}
& L \equiv\left\langle\tau^{2}\right\rangle^{1 / 2} U^{-1}, \\
& V \equiv\left\langle\left(\partial_{x} \psi\right)^{2}\right\rangle^{1 / 2}, \\
& D \equiv\left\langle\left(\partial_{x} \psi\right) \tau\right\rangle U^{-1},
\end{aligned}
$$

where $\psi$ and $\tau$ are the barotropic and baroclinic streamfunction, respectively, and the angle brackets refer to domain average. The diagnosed eddy scales (nondimensionalized by $U$ and $k_{d}$ ) are plotted in Fig. 2 as a function of $c_{D} k_{d}^{-1}$. The results of asymmetric drag are shown in red and the results of symmetric drag are shown in black. Different symbols indicate slightly different model settings with different domain sizes and resolutions (Fig. 1), which are examined to ensure the results are to a large extent not affected by the choice of these additional parameters with less physical interest. Also, we note that the asymmetric drag results are purposely plotted at $c_{D} k_{d}^{-1} / 2$ as opposed to $c_{D} k_{d}^{-1}$ for a reason that we explain shortly, and this does not change the interpretation of the scaling relations.

We first draw the attention to the asymmetric drag results. As shown in Fig. 2, all the eddy scales in the asymmetric drag simulations decrease with increasing $c_{D} k_{d}^{-1}$ when $c_{D} k_{d}^{-1}$ is small. When $c_{D} k_{d}^{-1}$ gets closer to $10^{0}$, this decreasing trend slows down, so the eddy scales can be described as exhibiting an exponential dependence over a limited range of damping. The case of $c_{D} k_{d}^{-1}=10^{0}$ seems to be an interesting transition point where both $L$ and $V$ reach their minimum. As $c_{D} k_{d}^{-1}$ continues to increase from $10^{0}, L$ and $V$ become larger again while $D$ still keeps decreasing. Here the bottom flows are strongly damped by friction and most kinetic energy is stored in the top layer. The strong asymmetry between the two layers led Arbic and Scott (2008) to argue that the theory based on barotropicbaroclinic decomposition is less relevant in this limit. They assumed instead that the inverse cascade occurs in the top layer and closed the theory with a diagnostic relation based on the primary balance in the bottom layer potential vorticity equation. These revised assumptions result in a new set of scaling laws, $D \sim c_{D}^{-1 / 5}$ and $L \sim c_{D}^{1 / 5}$. We find that it fits qualitatively to our simulations and apparently explains why the simulated $D$ and $L$ have opposite dependence on $c_{D} k_{d}^{-1}$. This reaffirms the crucial role played by layer asymmetry in this regime.

As a strong contrast, all the eddy scales in the symmetric drag simulations decrease monotonically when $c_{D} k_{d}^{-1}$ increases within the explored range. In the limit of small $c_{D} k_{d}^{-1}$, the symmetric drag results coincide with the asymmetric drag results. This is because most kinetic energy is barotropic and is equally partitioned between the two layers. The damping appearing in only the bottom layer is equivalent to the effective damping appearing in both layers but with a half of the damping strength (i.e., $c_{D} k_{d}^{-1}$ ). Hence, they are better aligned with each other when we shift the asymmetric drag results from $c_{D} k_{d}^{-1}$ to $c_{D} k_{d}^{-1} / 2$. As $c_{D} k_{d}^{-1}$ increases, unlike the exponential relation seen in asymmetric drag simulations, eddy scales for symmetric drag simply follow a power-law dependence on $c_{D} k_{d}^{-1}$ until $c_{D} k_{d}^{-1}$ 


\begin{tabular}{cccccc}
\hline \hline & Form of Friction & $k_{\max }$ & $k_{d}$ & IC & Damping Strength \\
\hline$\bigcirc$ & asym. quadratic & 128 & 50 & I & $10^{-3} \leq c_{D} k_{d}^{-1} \leq 10^{0}$ \\
$\bigcirc$ & sym. quadratic & 128 & 50 & I & $10^{-3} \leq c_{D} k_{d}^{-1} \leq 10^{0}$ \\
+ & asym. quadratic & 256 & 100 & I & $10^{-4} \leq c_{D} k_{d}^{-1} \leq 10^{-0.5}$ \\
+ & sym. quadratic & 256 & 100 & I & $10^{-4} \leq c_{D} k_{d}^{-1} \leq 10^{-0.5}$ \\
$\times$ & asym. quadratic & 128 & 10 & I & $10^{-1.5} \leq c_{D} k_{d}^{-1} \leq 10^{2}$ \\
$\times$ & sym. quadratic & 128 & 10 & I & $10^{-1.5} \leq c_{D} k_{d}^{-1} \leq 10^{2}$ \\
$\triangle$ & sym. quadratic & 128 & 10 & S & $10^{-1} \leq c_{D} k_{d}^{-1} \leq 10^{2}$ \\
$\square$ & asym. linear & 128 & 50 & I & $10^{-1} \leq \kappa k_{d}^{-1} U^{-1} \leq 10^{0}$ \\
$\square$ & sym. linear & 128 & 50 & I & $10^{-1} \leq \kappa k_{d}^{-1} U^{-1} \leq 10^{0}$ \\
\hline \hline
\end{tabular}

FIG. 1. A summary of the parameter settings for each set of numerical simulations: (from left to right) the chosen form of largescale friction, maximum wavenumber resolved $k_{\max }$, wavenumber of deformation radius $k_{d}$, initial condition (IC), and the explored range of nondimensionalized damping strength. For initial condition, "I" refers to the simulations integrated from the infinitesimal perturbations prescribed in the same way as LH95, and " $S$ " refers to the simulations integrated from the simulated streamfunction field taken from the $c_{D} k_{d}^{-1}=10^{-1}$ run that is integrated from the first initial condition to a statistically steady state.

reaches about $10^{0}$. Beyond this value, another powerlaw relation with a different slope is observed. The latter marks a new dynamical regime where the solution equilibrates without an inverse cascade. We analyze these simulations in more details in section 5 .

Based on the above results, we can now summarize the impact of layer asymmetry on eddy scaling into a few key findings. First, the asymmetry between the two layers as well as its impact is amplified when the damping is stronger. Second, the layer asymmetry is responsible for replacing a simple power-law scaling (as would have been predicted by the theory) with a more complicated transition between two different scaling regimes that in between could be approximated as exponential. Third, the layer asymmetry allows the inverse cascade to continue to occur in the upper layer even when $c_{D} k_{d}^{-1}$ exceeds one. After isolating the sources of complexity by comparing the asymmetric and symmetric drag simulations, we next examine the remaining complexities via comparing the symmetric drag simulations to the theoretical prediction by $\mathrm{H} 99$.

\section{Symmetric drag simulations versus theoretical prediction}

Without layer asymmetry, the two-layer system with symmetric drag seems more comparable to the two-dimensional system. It can therefore help better clarify the differences between the two systems that the theories of H99 and LH95 may have overlooked.
Looking at the symmetric drag results in Fig. 2, we notice that for $c_{D} k_{d}^{-1}<10^{0}$ the nondimensional characteristic scales of eddies can all be expressed as $\sim c_{D}^{-\mu}$. The exponent $\mu$ estimated from the least-squared fit using the data of $k_{d}=50$ simulations is indicated in Fig. 2. These estimates are smaller than predicted by Eqs. (3), (6), and (7): $L \sim c_{D}^{-0.58}, V \sim c_{D}^{-0.78}$, and $D \sim c_{D}^{-1.24}$ versus $L \sim c_{D}^{-1}, V \sim c_{D}^{-1}$, and $D \sim c_{D}^{-2}$ (we note that the wavenumber $c_{D}$ is inversely proportional to the length-scale $L_{D}$ ). The simulated eddy scaling dependence on the damping strength is consistently weaker than the theoretical prediction, so some assumptions in the theory must be violated.

To address this discrepancy, we believe a good starting point is to recognize that $L$ and $V$ scale differently with $c_{D} k_{d}^{-1}$. The fact that $L$ and $V$ are not proportional to each other contradicts an important implication by LH95, that is the eddy time scale $\left(T \equiv L V^{-1}\right)$ should scale with inverse Eady growth rate:

$$
T \sim\left(U k_{d}\right)^{-1} .
$$

Since $U$ and $k_{d}$ are fixed in each set of our simulations, Eq. (9) requires $L$ and $V$ to change proportionally if the theory of LH95 holds well. Equation (9) can be derived from Eqs. (2), (4), and (5) together with $\varepsilon_{c} \approx \varepsilon_{p}$. Using the definitions of $L$ and $V$ in Eq. (8), Eq. (9) can also be phrased as a constraint on the partitioning between barotropic kinetic energy $\left(\left\langle E_{\psi}\right\rangle \approx V^{2}\right)$ and baroclinic available potential energy $\left[\left\langle E_{\tau}\right\rangle=\left(L U k_{d}\right)^{2}\right]$ of isotropic eddies,

$$
\left\langle E_{\psi}\right\rangle /\left\langle E_{\tau}\right\rangle \sim \text { const }
$$

Evidently, because $L$ and $V$ change differently with $c_{D} k_{d}^{-1}$, this energy ratio varies and in fact increases along with $L$ in the simulations. A similar relation between $\left\langle E_{\psi}\right\rangle /\left\langle E_{\tau}\right\rangle$ and $L$ is documented by LH95 for their bottom linear drag simulations, where they also found that $\left\langle E_{\psi}\right\rangle /\left\langle E_{\tau}\right\rangle$ increases as energy containing wavenumber becomes smaller. They have connected this behavior to the observed shape of the energy spectra. In their simulations, they recognized that the slope of barotropic energy spectrum is slightly steeper than $-5 / 3$ and the slope of baroclinic energy spectrum is slightly flatter than $-5 / 3$ (cf. their Fig. 4). This deviation from Kolmogorov's theory is likely associated with a wide spread of energy injection from baroclinic to barotropic mode in wavenumber space. Taking the barotropic energy input's spectral nonlocality into account, they proposed a qualitative argument to explain the length-scale dependence of energy ratio. We have attempted to make this argument more quantitative to explain the weaker $c_{D}$ 

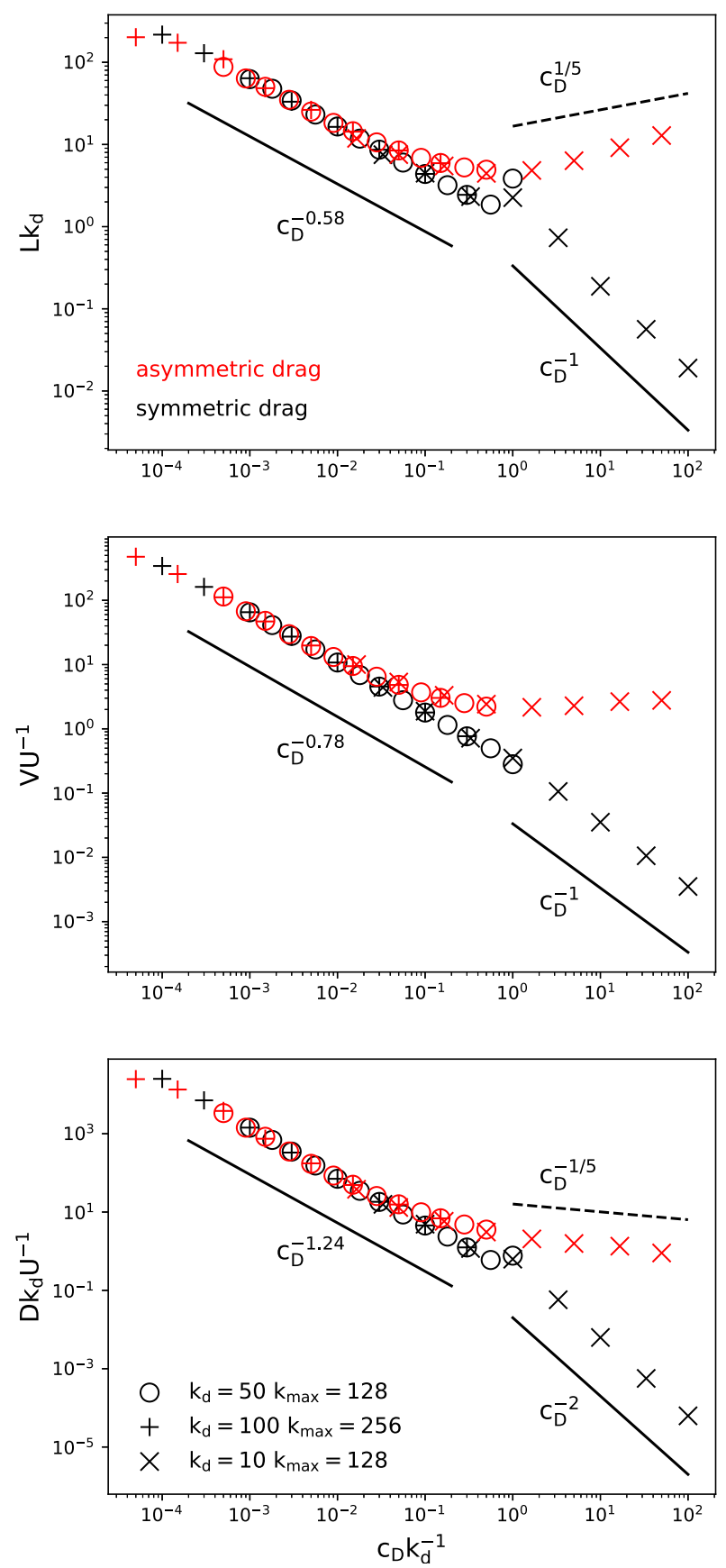

FIG. 2. Eddy scales in the asymmetric and symmetric drag simulations: (from top to bottom) eddy length scale $L$, velocity scale $V$, and diffusivity $D$ as a function of damping strength $c_{D} k_{d}^{-1}$. Simulations with the asymmetric drag are red and with the symmetric drag are black. The different symbols refer to the model settings with different domain sizes and spectral resolutions (see Fig. 1). For an easier comparison, all the asymmetric drag results are shifted by a factor of 2 along $x$ axis, i.e., from $c_{D} k_{d}^{-1}$ to $c_{D} k_{d}^{-1} / 2$ (which would be the effective drag if the drag was symmetric). In each panel, the top-left solid line indicates the power-law scaling estimated from a least-squared fit of the data in the $k_{d}=50$, $c_{D} k_{d}^{-1}<10^{0}$ symmetric drag simulations; the bottom-right solid dependence of eddy scales that is observed in our simulations.

To illustrate how this argument based on spectral nonlocality modifies the original scaling, we follow LH95's derivation to first obtain its modification to Eq. (9). The essence of this argument is to allow an underdetermined wavenumber $k$ dependence in barotropic energy spectrum $E_{\psi}(k)$. The theory of LH95 originally assumes that energy transfer from baroclinic to barotropic mode is confined at $k_{d}$ (Rhines 1977; Salmon 1978, 1980) so a Kolmogorov's inertial range exists and we expect $E_{\psi}(k) \sim \varepsilon_{\psi}^{2 / 3} k^{-5 / 3}$, where barotropic cascade energy flux $\varepsilon_{\psi}$ is independent of $k$. With the single dominant scale approximation, this directly leads to Eq. (2). However, if energy input is broadly spread among a range of wavenumbers and the local cascade energy flux picks up local energy injection as energy moves to smaller wavenumber, we may assume locally

$$
\varepsilon_{\psi}(k)=\varepsilon_{\psi 0} k_{d}^{x} k^{-x},
$$

with $x>0$ (we normalize $k$ by $k_{d}$ so that $\varepsilon_{\psi 0}$ has the unit of energy flux); the modified barotropic energy spectrum would therefore become

$$
E_{\psi}(k) \sim \varepsilon_{\psi 0}^{2 / 3} k_{d}^{2 x / 3} k^{-(5+2 x) / 3} .
$$

Then supposing that the baroclinic mode still acts like a passive tracer that is advected by the barotropic mode, the baroclinic energy spectrum would still follow $E_{\tau}(k) \sim \varepsilon_{\tau} T(k) k^{-1}$, where the baroclinic cascade energy flux $\varepsilon_{\tau}$ is constant and local eddy turnover time $T(k)$ is determined by the advection field. With the modified barotropic energy spectrum, $T(k) \sim\left[\varepsilon_{\psi 0}^{2 / 3} k_{d}^{2 x / 3} k^{2(2-x) / 3}\right]^{-1 / 2}$, the modified baroclinic energy spectrum becomes

$$
E_{\tau}(k) \sim \varepsilon_{\tau} \varepsilon_{\psi 0}^{-1 / 3} k_{d}^{-x / 3} k^{-(5-x) / 3} .
$$

Therefore, $x>0$ implies a steeper barotropic energy spectrum and a flatter baroclinic energy spectrum. An immediate result that follows this modification, supposing the bulk energies predominantly reside at a single wavenumber that is associated with $L$ and $\varepsilon_{\psi 0} \approx \varepsilon_{\tau}$, is that

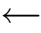

line indicates the power-law scaling predicted by H99's theory; and the top-right dashed line indicates the scaling proposed by Arbic and Scott (2008) in the strong asymmetric drag limit. 


$$
\left\langle E_{\psi}\right\rangle /\left\langle E_{\tau}\right\rangle \sim\left(L k_{d}\right)^{x},
$$

and the ratio between barotropic kinetic energy and baroclinic available potential energy should increase with $L$. With the definition of $T$, we obtain a modified scaling replacing Eq. (9):

$$
T \sim\left(L k_{d}\right)^{-x / 2}\left(U k_{d}\right)^{-1} .
$$

Compared with Eq. (9), Eq. (15) suggests $T$ is no longer a constant and depends on $L$ and $x$, which measures the broadness of energy input in the barotropic energy spectrum. Combining Eq. (15) with Eqs. (1), (4), and (5) gives the modified power-law dependence of eddy scales on $c_{D}$ :

$$
\begin{aligned}
& L \sim c_{D}^{-1 /(1+x)}, \\
& V \sim c_{D}^{-(2+x) /[2(1+x)]}, \\
& D \sim c_{D}^{-(4+x) /[2(1+x)]} .
\end{aligned}
$$

Hence, $x>0$ also implies a weaker $c_{D}$ dependence for $L, V$, and $D$.

To see if this modified scaling applies to our simulations, we estimate $x$ using Eq. (16) with the simulated scaling relation $L \sim c_{D}^{-0.58}$ to obtain $x \approx 0.72$. This estimate is then compared with the slopes of energy spectra to verify the self-consistency of the assumptions we have made on the spectral slopes. From Eqs. (12) and (13), we expect

$$
\begin{gathered}
{\left[E_{\psi}(k) k^{5 / 3}\right]^{3 / 2} \sim k^{-x},} \\
{\left[E_{\tau}(k) k^{5 / 3}\right]^{3} \sim k^{x}}
\end{gathered}
$$

near the energy-containing scale. These simulated spectra $\left\{\left[E_{\psi}(k) k^{5 / 3}\right]^{3 / 2}\right.$ and $\left.\left[E_{\tau}(k) k^{5 / 3}\right]^{3}\right\}$ are plotted in the first and second rows of Fig. 3. Although a single slope assumption does not fit to their shapes that well, $x \approx 0.72$ at least matches qualitatively to the observed bulk spectral slopes (for simplicity, only the set of $k_{d}=50$ simulations is shown).

In the third and fourth rows of Fig. 3, the simulated barotropic energy spectrum $E_{\psi}(k)$ and the simulated barotropic energy flux $\varepsilon_{\psi}(k)$ (calculated as described in LH95's Fig. 4) are further examined. To connect $E_{\psi}(k)$ back to $\varepsilon_{\psi}(k)$, we have compared the simulated $E_{\psi}(k)$ with the prediction $C_{\psi} \varepsilon_{\psi}(k)^{2 / 3} k^{-5 / 3}$ computed from the simulated $\varepsilon_{\psi}(k)$ and the universal constant $C_{\psi}=7$ chosen to give a reasonable fit. The latter is a good approximation for the former in the range of wavenumbers between the energy-containing scale (the peak of the spectrum) and $k_{d}=50$, confirming that energy level at these wavenumbers is solely determined by the local upscale cascade. The observed steepness of the spectral slope from $-5 / 3$ is then due to the $k$ dependence in $\varepsilon_{\psi}(k)$, which if to be approximated by $k^{-x}$ [i.e., Eq. (11)] is roughly consistent with the estimated $x \approx 0.72$.

Looking closely at the simulated $\varepsilon_{\psi}(k)$, we shall note that its spectrum is actually not so well described as $k^{-x}$. If all the energy is both input at a single wavenumber and then removed at a single smaller wavenumber, $\varepsilon_{\psi}(k)$ would be flat between these two wavenumbers. Instead, the actual spectral slope varies smoothly from negative to positive as $k$ decreases. Both barotropic energy input due to triad interactions involving the baroclinic mode, whose spectral structures look very similar to the ones reported in LH95 (cf. their Figs. 4 and 5), and the energy removal by quadratic frictional dissipation are nonlocal in wavenumber space, with the former dominant in the region of most interest, producing a negative slope. There is therefore no obvious physical basis for the use of the simple power-law approximation (i.e., $k^{-x}$ ) as compared to a more general functional shape that can better describe the $\varepsilon_{\psi}(k)$ spectrum. We emphasize that $k^{-x}$ in Eq. (11) is thus better regarded as a heuristic assumption that we choose for its simplicity and effectiveness to study the complexities that lead to the deviations from the original theoretical prediction.

We also note that in the above derivation for Eq. (16) we have ignored the change of the correlation between barotropic velocity and baroclinic streamfunction $[r \equiv D /(V L)]$ as a way to violate Eq. (4). From the estimated scaling fits (i.e., $\sim c_{D}^{-\mu}$ ) in Fig. 2, we can obtain $r \sim c_{D}^{0.12}$. The dependence of $r$ on $c_{D}$ could bring in uncertainty that leads to different estimates of $x$ when we fit the simulated $V$ and $D$ using Eq. (16) instead: $x \approx 0.79$ from simulated $V$ and $x \approx 1.03$ from simulated $D$. While this uncertainty range seems large for $x$ itself, it translates into much smaller ones in the estimated scaling exponents in Eq. (16) because of the specific way they depend on $x$. Its effect on the scaling argument is thus weak compared to the effects of the modified spectral slopes described above.

\section{Asymmetric drag simulations versus modified prediction}

When analyzing the spectra of the symmetric drag simulations, we have also checked the spectra of the asymmetric drag simulations. A finding from this analysis is that there is no obvious difference between the spectral slopes of the two sets of simulations (Fig. 3). This makes us suspect that the broad energy input in the barotropic energy spectrum remains as a key source of 

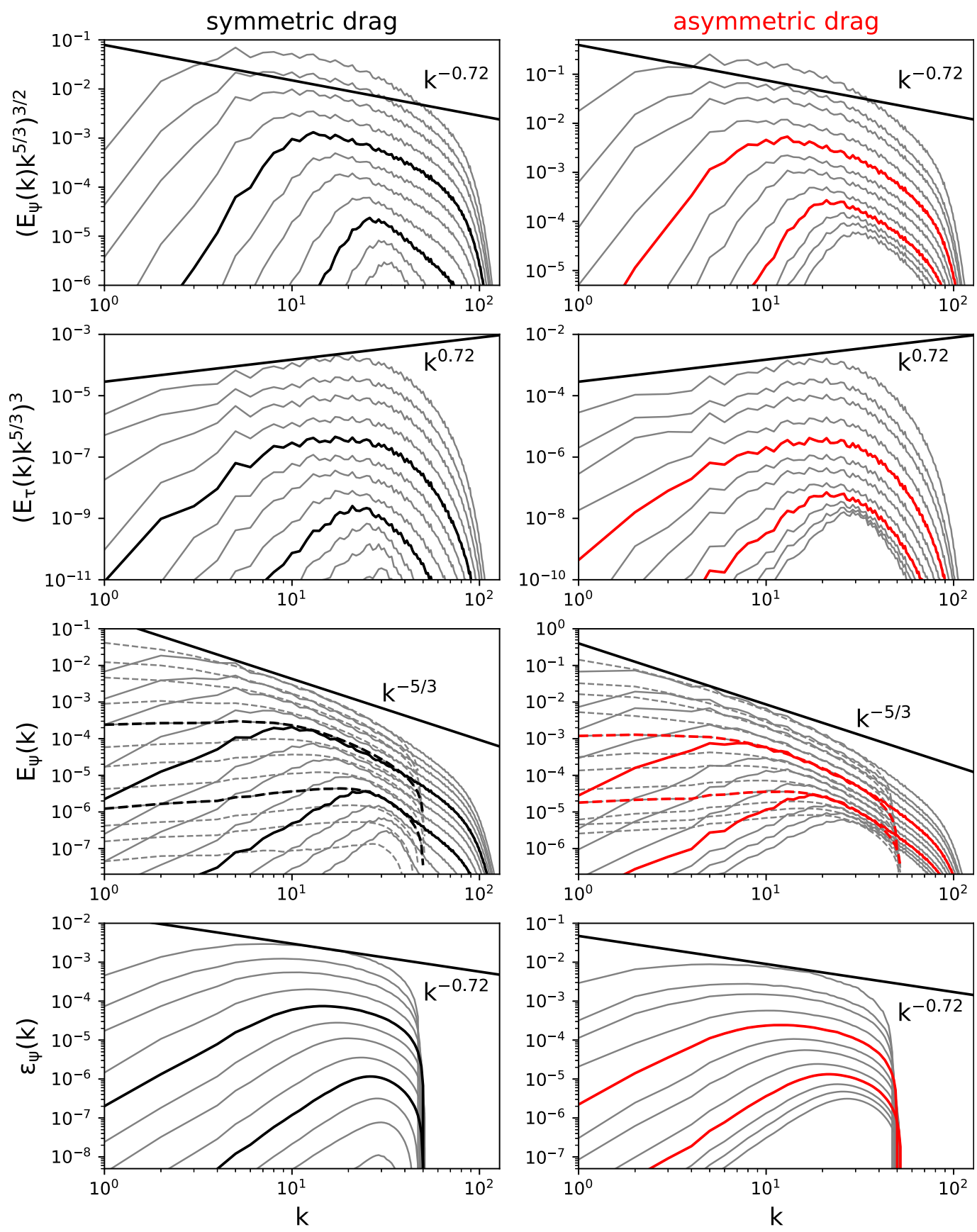

FIG. 3. Deviations of the simulated spectrum shapes from the theory in the (left) symmetric and (right) asymmetric drag simulations $\left(k_{d}=50\right.$ only): (from top to bottom) compensate spectra of barotropic energy $\left[E_{\psi}(k) k^{5 / 3}\right]^{3 / 2}$, compensate spectra of baroclinic available potential energy $\left[E_{\tau}(k) k^{5 / 3}\right]^{3}$, barotropic energy spectra $E_{\psi}(k)$ (solid) vs the corresponding predictions by $C_{\psi} \varepsilon_{\psi}(k)^{2 / 3} k^{-5 / 3}$ (dashed), and barotropic energy flux spectra $\varepsilon_{\psi}(k)$. In each panel, the spectra for the $c_{D} k_{d}^{-1}=10^{-1}$ and $c_{D} k_{d}^{-1}=10^{-2}$ simulations are colored, with the rest of the cases plotted in gray. We have chosen $C_{\psi}=7$ to obtain an overall reasonable fit for both sets of simulations, but we notice that this results in a slight overestimation for the symmetric drag simulations and a slight underestimation for the asymmetric drag simulations (for reasons unclear to us). The black solid line indicating the slope $k^{-x}$ or $k^{x}$ with $x \approx 0.72$ is estimated from the simulated $L$ using Eq. (16) (see text for more details). 
complexity in the asymmetric drag simulations and its effect may be largely separated from the effects of layer asymmetry. If we try to estimate $x$ in the modified scaling prediction for the asymmetric drag simulations, the resulted $x$ value is then hopefully similar to the one for the symmetric drag.

To test the hypothesis, we cannot estimate $x$ from Eq. (16). This is because Eq. (16) is derived from Eq. (1) and that Eq. (1) with $V$ defined in Eq. (8) does not hold with asymmetric friction. We instead estimate $x$ directly from Eq. (15), the scaling relation between $T$ and $L$, without a reference to $c_{D}$. The simulated $T$ itself turns out to be more sensitive to domain size and resolution than $L$ and $V$ individually, but their impact on the scaling is small. For simplicity, we only show $T$ for the sets of $k_{d}=50$ simulations in Fig. 4. Indeed, plotting $T$ against $L$ reveals that the asymmetric and symmetric drags behave more or less the same: $T$ scales with $L$ in a similar manner regardless the complexities of layer asymmetry. Fitting the data from both the asymmetric and symmetric results gives $T \sim L^{-0.36}$ and $x \approx 0.72$, which is consistent with the estimate from the symmetric results alone.

Considering that $T$ defined using $V$ in Eq. (8) may have little relevance when the asymmetry becomes too strong, we have also tried avoiding $V$ in the calculations by examining the simulated relation of $D$ and $L$ directly. As seen in Fig. 4, $D$ has a simple power-law dependence on $L$, and this dependence is very similar between symmetric drag and asymmetric drag simulations. A fit of the simulated $D$ on $L$ gives $D \sim L^{2.19}$. This result underscores that, while we cannot identified the relevant eddy velocity responsible for mixing in asymmetric drag simulations, at the very least $D$ itself scales as $L$ to some exponent that is close to (and thus can potentially be predicted by) the one for symmetric drag simulations.

Therefore, the moderate success of the modified scaling in predicting the symmetric as well as asymmetric drag results suggests the complexities due to the spectral nonlocality can be superposed on the complexities due to the layer asymmetry. The layer asymmetry affects the ability of drag to stop the cascade and results in different $L$ between asymmetric and asymmetric drag simulations, but it does not change the scaling relations between $L$ and other eddy statistics. Instead, the latter is primarily controlled by the effect of spectral nonlocality of barotropic energy input.

\section{Equilibration without an inverse cascade}

In this section, we investigate the distinct regime in the model with symmetric damping where the flows
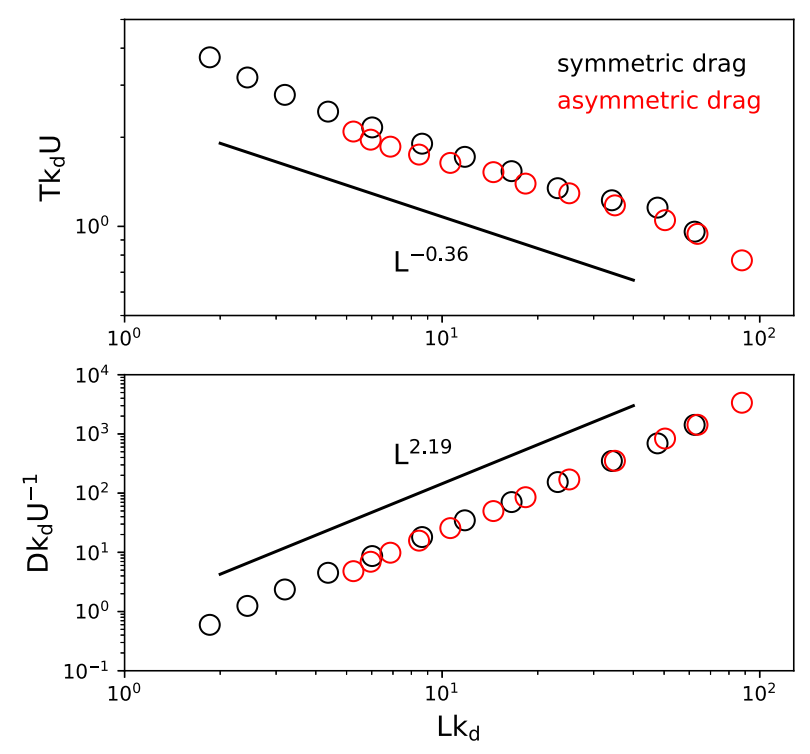

FIG. 4. Relations among eddy scales in the asymmetric and symmetric drag simulations ( $k_{d}=50$ only): relations of (top) eddy time scale $T$ and (bottom) eddy diffusivity $D$ with eddy length scale $L$. The slope of the black line is computed from the least squares fit of the data from both the asymmetric and symmetric drag simulations (i.e., all the data points shown in the plot).

equilibrate without an inverse cascade. The fact that there is no inverse cascade can be told from the results in Fig. 2 that $L k_{d}$ is about the same as or even smaller than $10^{\circ}$ in the symmetric drag simulations when $c_{D} k_{d}^{-1}>10^{0}$. However, some seeming contradictions appear in this finding. The disappearance of inverse cascade when $c_{D} k_{d}^{-1}>10^{0}$ is predicted by H99's theory, which argues that the friction becomes strong enough to directly stop the inverse cascade at the scale of deformation radius. Without an inverse cascade, Stone's (1972) quasi-linear theory predicts that eddy scales and amplitudes would be determined by the properties of linear unstable modes and the given basic state. Therefore, they should be independent of the frictional strength. In these simulations, $L$, the meridional length scale of eddy mixing, can nonetheless become much smaller than $k_{d}$ when the friction strengthens, and $V$ and $D$ can also be smaller than $U$ and $U k_{d}^{-1}$, respectively. Surprisingly, the eddy scaling in this regime matches the theoretical prediction by H99 [i.e., Eqs. (3), (6), and (7)]. We here explain how these counterintuitive results emerge by examining the equilibration mechanism of these flows.

To study how the two-layer system equilibrates with a strong symmetric quadratic drag, we have taken a close look at the instantaneous streamfunction in these simulations. The simulated streamfunction has no meridional structure and tends to lock to certain zonal 
wavenumbers, indicating that the flows are dominated by the baroclinically unstable modes with gravest meridional wavenumber rather than isotropic eddies. This also suggests that it is the friction alone that directly stops the unstable modes from continuing to grow. The nonlinear terms and secondary instabilities do not have a chance to emerge so as to modulate the meridional scale of eddies and make it comparable to the zonal scale.

In addition to the instantaneous streamfunction fields, we can also see the dominance of meridional velocity $v$ over zonal velocity $u$ in the eddies and the dominance of baroclinic over barotropic component in the kinetic energy partitioning (Fig. 5). Consequently, the eddy fluxes can no longer be thought of as barotropic flows stirring baroclinic streamfunction (as in H99). It is also less meaningful to try to relate the zonal energy containing scale to meridional mixing because the zonal scale has no direct relation to the meridional scale. While the zonal eddy scale is controlled by the wavelength of linear unstable modes, the assumption that eddies would be isotropic, which is implicitly included in Stone (1972), does not apply to these simulations. Thus, Stone's (1972) theory is inapplicable here.

What the linear dynamics of baroclinic instability controls here is instead the baroclinic eddy time scale $\left(T_{\tau} \equiv L / V_{\tau}\right)$ defined by baroclinic meridional velocity $V_{\tau}$. This time scale represents the inverse linear growth rate of the system's gravest unstable modes. Equating $T_{\tau}$ to the time scale of frictional damping $\left(c_{D} V_{\tau}\right)^{-1}$ yields $L \sim c_{D}^{-1}$, which leads to a recovery of Eq. (3). If we further consider that baroclinic kinetic energy is proportional to the barotropic kinetic energy in baroclinic waves, $V_{\tau} \sim V$, then we can follow the same derivation described in section 1 to regain Eqs. (6) and (7). Therefore, the eddy scaling in this regime is the same as the H99's prediction because of the same time-scale constraint but with the eddy time scale determined by linear instability rather than turbulent cascade.

Additionally, it is worth noting that since the flows equilibrate directly with the gravest unstable modes, the equilibrated eddy statistics are sensitive to initial conditions. The simulations analyzed above are all integrated from the infinitesimal perturbations and go through the same linear baroclinic growth stage, so the excited modes and $T_{\tau}$ in these simulations are the same (Fig. 5). To demonstrate the initial-condition dependence, we have integrated another set of simulations with varying $c_{D}$. These new simulations are initiated with the streamfunction field at the last output time step of the $c_{D} k_{d}^{-1}=10^{-1}$ simulation integrated from infinitesimal perturbations until statistically steady state (i.e., from the set of simulations
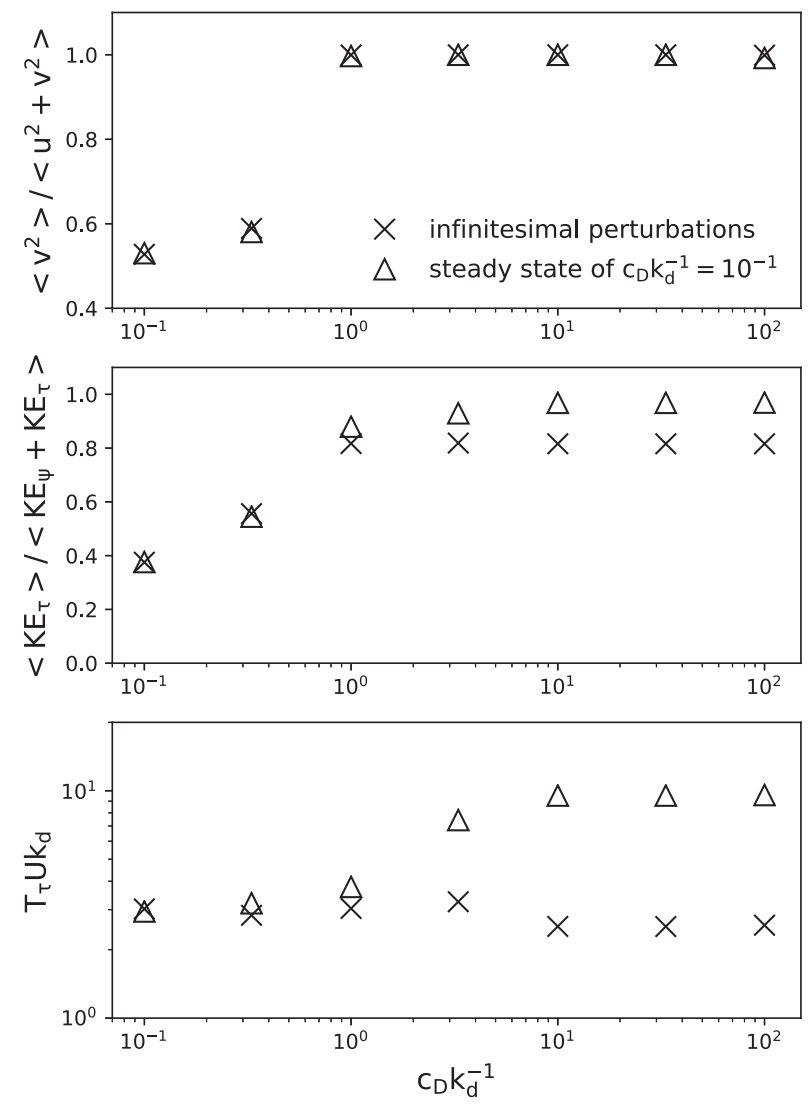

FIG. 5. Initial-condition dependence of steady state flow properties in the strong symmetric drag regime $\left(k_{d}=10\right.$ only): (from top to bottom) meridional kinetic energy $\left(\left\langle v^{2}\right\rangle / 2\right)$ to total kinetic energy $\left(\left\langle u^{2}+v^{2}\right\rangle / 2\right)$ ratio, baroclinic kinetic energy $\left(\left\langle\mathrm{KE}_{\tau}\right\rangle\right)$ to total kinetic energy $\left(\left\langle\mathrm{KE}_{\psi}+\mathrm{KE}_{\tau}\right\rangle\right)$ ratio, and baroclinic time scale ( $T_{\tau}$ defined as the ratio of eddy length-scale $L$ and baroclinic meridional velocity $V_{\tau}$ ) as a function of $c_{D} k_{d}^{-1}$. Two initial conditions are considered: one with infinitesimal perturbations in the streamfunction field as described in LH95 (crosses) and the other as the steady-state streamfunction field taken from the $c_{D} k_{d}^{-1}=10^{-1}$ simulation (triangles).

analyzed in Fig. 2). They are then integrated until the new statistically steady state is achieved. During this transition, the flows initially occupied by isotropic eddies and filaments gradually spin down and are eventually taken over by the unstable modes. Therefore, the meridional-to-zonal kinetic energy ratio is still close to one. However, the unstable modes emerged at the statistically steady state are different, in general, so the ratio of baroclinic-to-barotropic kinetic energy and $T_{\tau}$ exhibit sensitivity to initial conditions (Fig. 5).

\section{Linear versus quadratic drag}

While our analysis focus on the simulations with the more geophysically relevant quadratic drag, it is 
the linear drag that is more commonly used in previous studies of this type. To better link our results to this literature, we have conducted additional simulations with linear drag. We plot the diffusivity $D$ diagnosed from these linear drag simulations along with the ones from the quadratic drag simulations in Fig. 6. The two set of simulations share the identical setting except for the form of drag. The key distinction of a linear drag from a quadratic drag is that it defines a time scale rather than a length scale. Its strength is measured by the inverse damping time scale ( $\kappa$ as defined in LH95). To allow a straightforward comparison between two forms of drag, we define an effective damping time scale $\kappa_{e} \equiv c_{D}\left|\nabla \psi_{2}\right|$ for the quadratic drag simulations, where $\left|\nabla \psi_{2}\right|$ is the bottom-layer rms velocity horizontally averaged over the domain (an internal scale diagnosed from the simulations). With this definition, the results of quadratic and linear collapse to a single line in Fig. 6, indicating they share the same eddy-scale dependence on damping strength.

The possibility to map the linear to quadratic drag simulations further suggests that the scaling relations and theoretical consideration in this study may also help explain the dependence on linear drag, such as why the linear drag itself can stop the cascade alone (Smith and Vallis 2002). If we take the theory with the modified spectral slopes described in section 3 , but in addition use the approximation $c_{D} \sim \kappa V^{-1}$ in the expression for $V$ in Eq. (16), we get

$$
V \sim \kappa^{-(2+x) / x}
$$

As expected, this is indeterminate for $x=0$ (the H99 scaling) but is well defined for $x>0$. Substituting this scaling for $V$ into the expression for $L$ and $D$, we find

$$
\begin{aligned}
& L \sim \kappa^{-2 / x}, \\
& D \sim \kappa^{-(4+x) / x} .
\end{aligned}
$$

For $x \approx 1.03$ estimated from the $D$ of symmetric quadratic drag simulations in section 3, this implies $D \sim \kappa^{-4.88}$. This scaling is indicated with the sloping line in Fig. 6 and is close to the least-squared estimate obtained from the symmetric linear drag simulations, $D \sim \kappa^{-4.52}$. The reasonable fit to the simulated results suggests that this way of describing the results in terms of a modified spectral slope is consistent between the simulations with two forms of drag.

There is however a particular aspect of which the linear drag acts differently from the quadratic drag. While in strong symmetric quadratic drag simulations the eddies equilibrate linearly, they are damped to zero

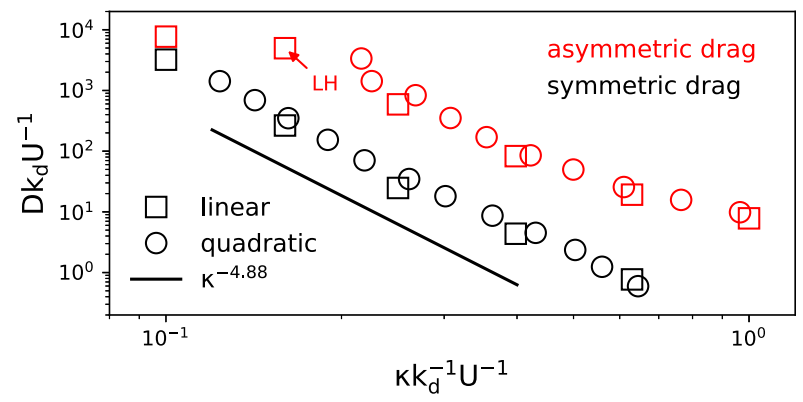

FIG. 6. Eddy scales in the linear and quadratic drag simulations: diffusivity $D$ diagnosed from linear (squares) and quadratic (circles) drag simulations as a function of linear damping strength $\left(\kappa k_{d}^{-1} U^{-1}\right)$. The two sets of simulations are mapped to each other by comparing $\kappa$ to the effective linear damping strength $\kappa_{e}$ (defined in the text) for the quadratic drag simulations. $\mathrm{LH}$ denotes for the run that shares the same parameter setting as the case I simulation in LH95. The solid line indicates the scaling prediction discussed in the text.

in strong symmetric linear drag simulations when the inverse damping time scale is larger than the growth rate of the most unstable modes. (With linear potential vorticity damping, i.e., with equal damping of winds and temperature, stabilization occurs precisely when the damping is larger than the growth rate of most unstable inviscid mode, but equal frictional damping in both layers with no thermal damping is sufficient to produce the same qualitative result.) In contrast, quadratic damping can never stabilize a flow that is unstable in the absence of the damping, since the strength of the damping is infinitesimal when the wave is infinitesimal. It only interferes with the baroclinic instability once the wave grows sufficiently that the damping time scale and the time scale of baroclinic growth become comparable.

\section{A comparison with TY06}

The modified scaling argument present in this study shares with LH95 the same physical picture for eddy heat fluxes, which are interpreted as generated by the stirring of background mean temperature (baroclinic streamfunction) gradient by the barotropic energy-containing eddies. We have proposed that focusing on the spectral shapes and their departure from Kolmogorov's scaling is a useful way of obtaining modified scaling to fit our simulations, with quadratic symmetric, quadratic asymmetric, linear symmetric, and linear asymmetric drags. However, its relevance to the linear asymmetric drag case is confusing given the findings in TY06, where the eddy heat fluxes are described as due to the systematic meridional drift of coherent vortices carrying temperature signatures. 

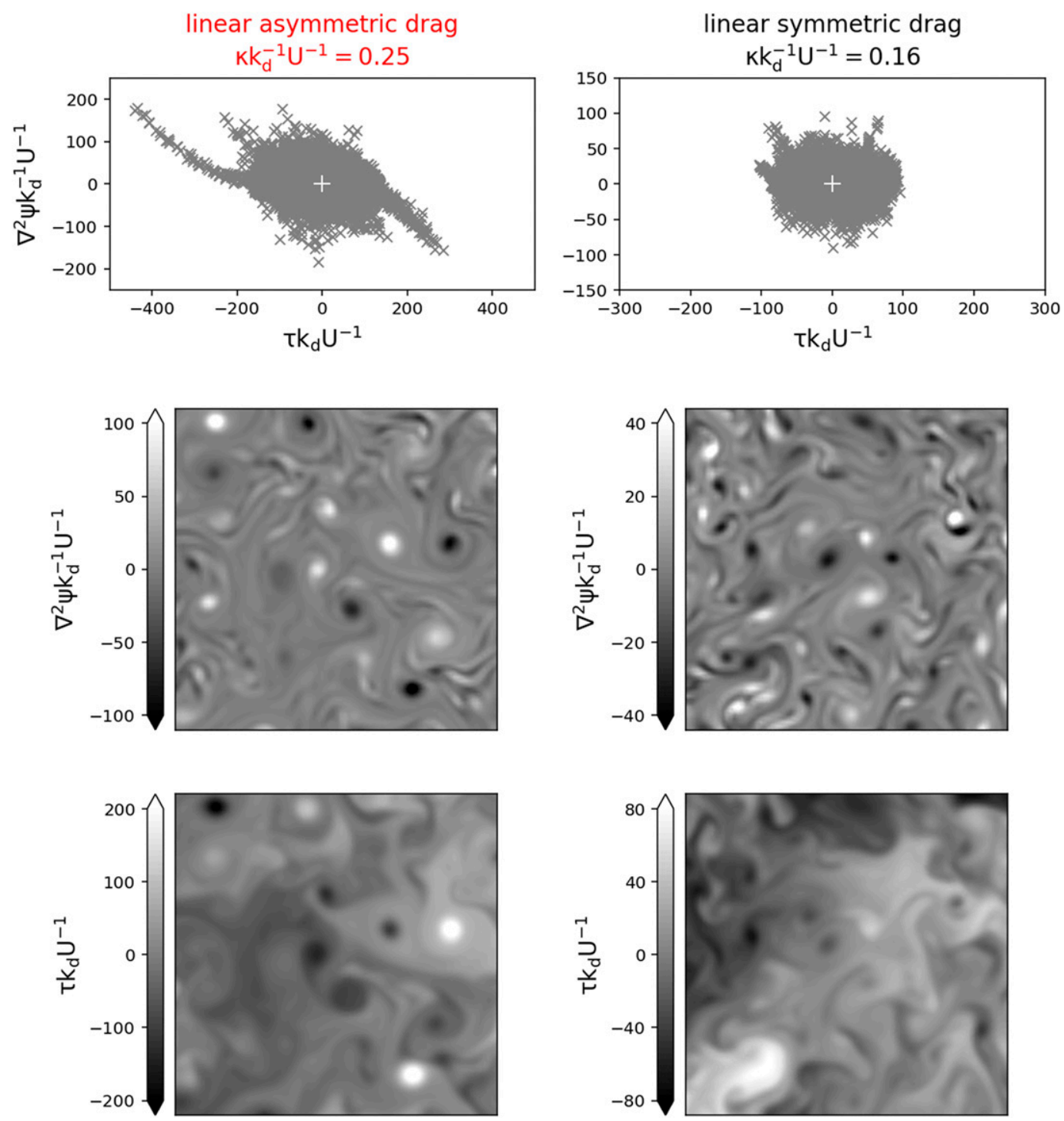

FIG. 7. (top) Scatterplots of baroclinic streamfunction $\tau$ and barotropic vorticity $\nabla^{2} \psi$ and (middle),(bottom) snapshots of the two fields: (left) a simulation using linear asymmetric drag with $\kappa k_{d}^{-1} U^{-1}=0.25$ and (right) another using linear symmetric drag with $\kappa k_{d}^{-1} U^{-1}=0.16$ are shown to compare with the simulations of TY06.

In TY06's analyses, they described a strong anticorrelation between the instantaneous baroclinic streamfunction $\tau$ and barotropic vorticity $\nabla^{2} \psi$ (cf. their Fig. 4) to emphasize this role of vortices in heat transport. Similar to those shown in TY06, in Fig. 7, we present scatterplots of $\tau$ and $\nabla^{2} \psi$ (normalized by $k_{d}$ and $U$ ) along with the snapshots of the two fields that are used to generate these scatterplots (to allow the finer structure in the snapshots to be recognized more easily, only one-ninth of the entire domain is shown). We have examined all our simulations and have selected two of them as examples for this purpose. The two selected simulations are both with linear damping: the one with asymmetric drag has $\kappa k_{d}^{-1} U^{-1}=0.25$ and the one with symmetric drag has $\kappa k_{d}^{-1} U^{-1}=0.16$. We choose the former as it is among our simulations most comparable to the simulation shown in Fig. 4 of TY06. Accordingly, we then choose the later case that uses symmetric drag and has similar eddy scales (e.g., the simulated $D$ for the two cases are similar as shown in Fig. 6).

For the asymmetric drag case, we see a scatterplot similar to the one shown in TY06 with the data partly organized into a few branches that correspond to individual vortices. It also confirms the presence of the anticorrelation between $\tau$ and $\nabla^{2} \psi$ as identified by TY06. When we look at the scatterplot of the symmetric drag 
case, there is however no evidence that $\tau$ and $\nabla^{2} \psi$ are correlated. The disappearance of the anticorrelation does not imply the disappearance of the coherent vortices, though. For both simulations, we have observed a hierarchy of vortices with different sizes in the snapshots of $\nabla^{2} \psi$ that look indistinguishable. Only when turning to the $\tau$ field, their differences start to be revealed. For the asymmetric drag case, the vortex structures are vivid in $\tau$, with the warm core anticyclonic signature expected. By contrast, it is more difficult to identify the vortices in the symmetric drag case's $\tau$ field. Even if they exists, the sign of $\tau$ and $\nabla^{2} \psi$ at their cores is not systematic. Instead, there is a cluster of data around the origin in the $\tau-\nabla^{2} \psi$ scatterplots, corresponding to the long thin filaments occupying most of the domain and seen in both $\nabla^{2} \psi$ and $\tau$ fields. In this regard, a nonnegligible portion of $\tau$ anomalies is not within the vortices but is within the filaments aroused from the baroclinic forward cascade. One key to reconcile these two viewpoints would therefore be an understanding of the partitioning of the heat transport between vortices and passive filaments.

The simplest explanation for the thermal structure of the vortices described in TY06 is that the lowerlevel winds in the vortices that would otherwise be barotropic are damped by friction, creating top-heavy equivalent barotropic vortices. Consistent with thermal wind, this results in warm-core anticyclones and cold-core cyclones. Given these thermal signatures, the vortex dynamics in the presence of the mean thermal wind becomes a candidate for organizing heat transfer, in competition with the passive tracer-like advection of the temperature field. Yet, in the case of symmetric damping, there is no reason to expect this kind of equivalent barotropic structure. By symmetry, the eddy kinetic energies in the two layers must have identical statistics in this case. This may explain why we only observe the anticorrelation of $\tau$ and $\nabla^{2} \psi$ in the asymmetric but symmetric drag case. Moreover, in our results, the scaling relationships for diffusivity and the eddy scales look identical in the symmetric and asymmetric drag cases in the limit of weak damping (Figs. 2 and 6). If the vortices in the symmetric drag cases can have no systematic temperature signal, a vortex-dominated heat flux is difficult to visualize. By implication, if we plausibly want the very similar results with asymmetric drag to have the same physical explanation as in the symmetric drag case, it seems justified to consider both as dominated by the same dynamics when damping is weak.

For stronger damping, there are clearly important differences between the symmetric and asymmetric drag cases (Figs. 2 and 6). When the nondimensional damping strength is larger than one, the flows are at another limit described in Arbic and Scott (2008) and Arbic and Flierl (2004). Our current speculation is that TY06 dynamics is relevant for intermediate values of the damping strength in the asymmetric drag case, interpolating between that limit of strong damping and the weak damping regime for which the scaling relations discussed here are relevant. (We restrict our discussion to cases in which the domain size is not a relevant parameter.) This intermediate regime is very important, being in a meteorological and oceanic relevant parameter range (H99; Arbic and Scott 2008). The analysis in TY06 likely provides more insights to the dynamics in this regime, even though we cannot at this time offer a coherent theory that simultaneously covers all of these regimes.

\section{Homogeneous and inhomogeneous comparison}

It is natural to question how these homogeneous turbulence results carry over to inhomogeneous flows. As the first step, we have explored an inhomogeneous two-layer quasigeostrophic model with channel geometry. The equation of the model is

$$
\frac{\partial q_{i}}{\partial t}+J\left(\psi_{i}, q_{i}\right)=-\kappa_{T}(-1)^{i} \frac{\left(\tau-\tau_{E}\right)}{\lambda^{2}}+\delta_{i 2} \mathscr{D}_{i}\left(c_{D}\right)-\nu \nabla^{6} \psi_{i}
$$

where $q_{i}=\nabla^{2} \psi_{i}+(-1)^{i}\left(\psi_{1}-\psi_{2}\right) /\left(2 \lambda^{2}\right)$ is potential vorticity and $\psi_{i}$ is streamfunction for upper $(i=1)$ and lower $(i=2)$ layers; $\mathscr{D}\left(c_{D}\right)$ represents the quadratic drag in the same form as Eq. (A4) and $\nu \nabla^{6} \psi$ is the hyperviscosity term. Except the hyperviscosity term, the inhomogeneous model is only distinct from the homogeneous model in that its baroclinic streamfunction $\left[\tau=\left(\psi_{1}-\psi_{2}\right) / 2\right]$ is relaxed to a zonally symmetric profile that produces a baroclinic jet. As this thermal relaxation is controlled by the inverse thermal damping time scale $\kappa_{T}$ and the thermal equilibrium profile $\tau_{E}$, we can construct a diffusive model (in the direction $y$ ) of the form

$$
\frac{\partial \tau(y)}{\partial t}=\frac{\partial}{\partial y}\left[D_{e}(y) \frac{\partial \tau(y)}{\partial y}\right]-\kappa_{T}\left[\tau(y)-\tau_{E}(y)\right],
$$

where $D_{e}$ is the effective dimensional diffusivity in the form $D_{e}(y)=-\partial_{y} \tau(y) \lambda d$ and $d$ is the nondimensional diffusivity measured from the homogeneous model. That is, given that the two models have the same nondimensional frictional damping strength (i.e., $c_{D} k_{d}^{-1}=$ $c_{D} \lambda$ ), we can obtain $d=D k_{d} U^{-1}$ from the simulated diffusivity $D, k_{d}$, and $U$ in the corresponding homogeneous model simulation to solve Eq. (22) for the equilibrated temperature and eddy heat flux. 

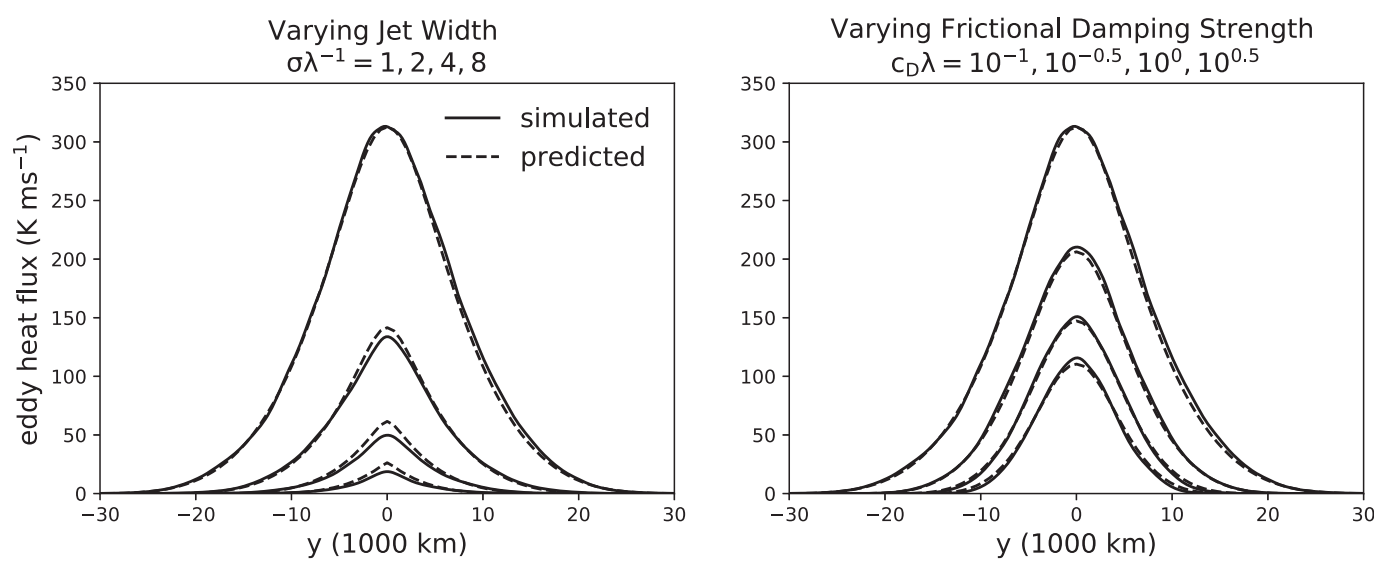

FIG. 8. Eddy heat fluxes in the channel model simulations: the meridional structure of eddy heat fluxes simulated from the channel model [Eq. (21); solid] and the predictions by a diffusive energy balance model with the diffusivity predicted by our homogeneous model simulations [Eq. (22); dashed]. Two sets of simulations are examined: one with varying jet width at radiative equilibrium $\sigma$ and one with varying frictional quadratic damping strength $c_{D}$. (left) The first set of simulations has a fixed $c_{D} \lambda=10^{-1}$ and $\sigma \lambda^{-1}=1,2,4,8$; (right) the second set of simulations has a fixed $\sigma \lambda^{-1}=8$ and $c_{D} \lambda=10^{-1}, 10^{-0.5}, 10^{0}, 10^{0.5}$, where $\lambda$ is deformation radius. The other model parameters is described in the text.

Figure 8 shows how the eddy heat fluxes predicted by this diffusive model compare to the inhomogeneous model simulations with the parameters $\beta=0$, $\lambda=\sqrt{2}^{-1} 700 \mathrm{~km}, \kappa_{T}^{-1}=20$ days, $\tau_{E}=-U_{E} \sigma \tanh (y / \sigma)$, and $U_{E}=20 \mathrm{~m} \mathrm{~s}^{-1}$. These are chosen to be mostly the same as in Zurita-Gotor et al. (2014), and the eddy heat fluxes are also shown in the temperature $(\theta)$ unit $(\mathrm{K})$ through the conversion $\theta \equiv \tau \psi_{0}^{-1} \theta_{0}$ with $\theta_{0}=$ $290 \mathrm{~K}$ and $\psi_{0}=2.45 \times 10^{8} \mathrm{~m}^{2} \mathrm{~s}^{-1}$. The inhomogeneous model's resolution and domain size in the zonal direction are also kept the same as the homogeneous model after nondimensionalized. This is to ensure the results are not affected by these model details. Evidently, the diffusive model successfully predicts the eddy heat flux's meridional structure both when the radiative equilibrium width of baroclinic jet $\sigma$ and when the frictional (quadratic) damping strength $c_{D}$ are varied.

This comparison has previously been provided by Pavan and Held (1996) with nonzero $\beta$ and linear damping (cf. their Fig. 9). Our case with zero $\beta$ is even simpler in the sense that there is no ambiguity whether to diffuse temperature or potential vorticity, since the temperature and the potential vorticity in both layers are all proportional to each other in this limit of the homogeneous model. In the inhomogeneous model simulations we analyzed, the eddy momentum fluxes are also negligible. Consistent with Pavan and Held (1996), our result again supports the relevance of homogeneous scaling for inhomogeneous flows. We note that, as in Pavan and Held (1996), thermal damping has been added to the homogeneous model when calculating $d$ as this is an essential feature of how the inhomogeneous model is forced. Since the thermal damping is not included in the homogeneous simulations analyzed in the previous sections, the $d$ obtained from the homogeneous simulations with thermal damping in general is different from those without thermal damping and reported in Fig. 2. In the case considered here, it has a very modest effect on the eddy scales and fluxes, so $d$ remains close to the value that we have shown there. Yet some systematic deviations from the homogeneous predictions, possibly due to this extra parameter or other subtle differences between the two models, are under investigation.

\section{Discussion and conclusions}

We have studied the statistically steady state solutions of a $\beta=0$ homogeneous two-layer quasigeostrophic model with quadratic drag. In this model, the statistically steady state is a function of a single parameter, the ratio of a frictional length-scale $L_{D}=C_{D}^{-1} H$ (where $C_{D}$ is the nondimensional coefficient in the surface stress and $H$ is the depth of the layer to which the stress applied) and the radius of deformation $\lambda=N H / f$, or, equivalently in terms of our model notations, the ratio of their wavenumbers: $c_{D} k_{d}^{-1}$. We have run the model with a range of $c_{D} k_{d}^{-1}$ in the attempt to understand the aspects of its dynamics that complicate the eddy scaling theory proposed by H99.

The first aspect we focus on are the asymmetry between upper and lower layers caused by the presence of drag in the lower layer only. This layer asymmetry in 
drag makes the flow equivalent barotropic. Therefore, when the damping is stronger, while both asymmetric and symmetric drag become more efficient at stopping the inverse cascade, the former is relatively more inefficient than the latter. Eddy scales are then decreasing less slowly with the damping strength in the cases with asymmetric than with symmetric drag. On the other hand, when the damping is weak and the flow is largely barotropic, there is little difference between models with asymmetric or symmetric drag, as long as one rescales the strength of the damping so that the barotropic flow is damped at the same strength in each case.

In the second aspect, we argue that the spectrally nonlocal baroclinic-to-barotropic energy transfer results in the deviation from Kolmogorov's inertial range theory, which is a central assumption in LH95 that is then considered in H99. A modest modification on this assumption and then H99's scaling by assuming barotropic spectral energy flux has a wavenumber dependence $k^{-x}$ is adopted to explain the observed weaker powerlaw dependence of eddy scales on the damping strength $\left(L \sim c_{D}^{-0.58}, V \sim c_{D}^{-0.78}\right.$, and $\left.D \sim c_{D}^{-1.24}\right)$. We do not provide a theory for $x$, but find that this expression, with $x \approx 0.72$ directly estimated from the simulations, gives self-consistent fits to the simulations, although some uncertainty exists on how it is best estimated. We also confirm that the same modified scaling applies to both the symmetric and asymmetric drag simulations when the damping is weak, so the complexity related to the spectral nonlocality can be largely distinguished from the one related to the layer asymmetry. Finally, with sufficiently strong symmetric damping a regime is found in which the equilibrated flows are dominated by the linear unstable modes directly stabilized by friction, so the linear dynamics controls the eddy scaling and the solutions are initial-condition dependent.

In addition to quadratic drag, we examine the homogeneous model simulations with linear drag and find that their eddy scales are very similar to those in the quadratic drag simulations once a proper relation between the damping strengths of the two forms of drag is defined. Because of this simplicity, the modified scaling for the quadratic drag case also predicts the scaling for the linear drag case, which resolves the problem of the original theories of LH95 and H99 that struggle to provide a well-defined prediction when the halting mechanism is linear drag. After that, we discuss the alternative approach of TY06, in which heat transport in their bottom linear drag simulations is found to be due to the drift of vortices with systematic temperature signatures. This is also observed in our asymmetric drag simulations. However, the similarity in the behavior of our model with symmetric and asymmetric drags when the damping is weak makes it hard to interpret our results in this fashion, since the model with symmetric drag produces vortices with no systematic temperature signals. Therefore, we leave this issue unresolved.

Last, following the approach of Pavan and Held (1996), we compare a set of inhomogeneous channel model simulations with bottom quadratic drag to the solution of a diffusive energy balance model with its diffusivity predicted by the homogeneous model. At least for the cases that we have looked into, we obtain some encouraging results. In fact, with $\beta=0$, this is an especially simple system to perform such a comparison, in large part because eddy momentum fluxes play less of a role here than in the case with nonzero $\beta$, resulting in the absence of eddy-driven jets. Further study of this comparison in this idealized system could shed light on any fundamental limitations to the value of the homogeneous limit.

Together these results help us to better interpret H99's estimate on the extent of inverse cascade controlled by surface friction in idealized models of the extratropical troposphere. Relative to Eq. (3)'s prediction, accounting for spectral nonlocality helps the cascade stop earlier, while introducing layer asymmetry allows a larger cascade range. The two counteracting effects both operate at $L_{D} \lambda^{-1}=(f / N) C_{D}^{-1} \approx 10$, the relevant regime for the extratropical troposphere over oceans $\left(f / N \approx 10^{-2}\right.$ and $\left.C_{D} \approx 10^{-3}\right)$, so Eq. (3) ends up being a fair estimate. If a higher surface roughness, such as for the typical land surface value $\left(C_{D} \approx 10^{-2}\right)$, is considered, the prediction by Eq. (3) may underestimate the eddy length scale at which the surface friction halts the cascade. The eddy kinetic energy and diffusivity may also be larger than expected. As it is a meteorological relevant regime, the effects of friction being confined near the surface clearly need a better quantification for a more precise estimate. This is not addressed here and is a limitation of this study.

Another limitation we emphasize is that the modified scaling presented in this study is not a predictive theory but rather an economical description, as we directly estimate the wavenumber dependence of the barotropic spectral energy flux (i.e., $k^{-x}$ ) from the simulations. To understand how this flux is determined, one would have to study the triad interactions and the associated spectral energy transfers into the barotropic mode. Added on these uncertainties are also the somewhat fuzzy connection assumed in LH95 between the spectrally local and spectrally integrated properties, 
where the assumption that the energy containing eddies producing the eddy heat flux can be described by a single wavenumber (Smith and Vallis 2002), and the variations of correlation between the barotropic velocity and baroclinic streamfunction in the mixinglength theory. Still, we are convinced that this modification of LH95's scaling may capture some of the important physics. The exponent of the simulated power-law relation is reasonably well defined and the spectral slope discrepancy between the simulations and theory is qualitatively consistent between our simulations and those reported by LH95. It at the very least helps us to better connect the understanding of two-layer baroclinic turbulence and of barotropic turbulence.

These baroclinically unstable homogeneous models are also potentially relevant to the ocean mesoscale eddy parameterization problem. In this regard, it is useful to compare our results to those in Jansen et al. (2015). They have parameterized the eddy heat fluxes in a two-layer primitive equation channel model using an empirical fit to relate barotropic and bottom kinetic energies as a key assumption. In the limit of $\beta=0$, they use the constant eddy time-scale assumption proposed by LH95 [i.e., Eq. (9)] to form a closure. Their scheme takes into account layer asymmetry due to the bottom drag (and the different layer depths) but assumes no further adjustments to LH95's scaling. This assumption may be adequate for parameterizations in which there are many other uncertainties, but the constant eddy time scale in LH95 is not supported by our results. Jansen et al. (2015) has also found some issues with LH95's scaling when nonzero $\beta$ is included. There are a number of results in the literature, using a variety of models, that differ regarding the utility of Held and Larichev (1996) theory, the extension to nonzero $\beta$ of the LH95's scaling (Smith and Vallis 2002; Barry et al. 2002; Lapeyre and Held 2003; Zurita-Gotor 2007; Thompson and Young 2007; Jansen and Ferrari 2013; Jansen et al. 2015; Chai 2016). A natural question is then how much of the mismatch to LH95's theory with nonzero $\beta$ can be attributed to deficiencies that are already present in the absence of $\beta$. Building on the results presented here, we will address parts of this question in a separate study.

In the context of studies on eddy scaling in the homogeneous two-layer quasigeostrophic model, this work resides at the simplest extreme on the model hierarchy. In fact, we have taken an extra step backward to investigate a more unrealistic model with mechanical drag appearing symmetrically between the two layers. This even simpler model configuration, in which all eddy statistics are identical in the two layers, offers the potential for a cleaner comparison between a self-stirred baroclinically unstable flow and the stirred barotropic model. By studying this limit, we have emphasized knowledge gaps between baroclinic and barotropic turbulence. We believe that this is a relevant limit in which to try to solidify our understanding of eddy statistics in these turbulent baroclinically unstable flows and to provide a more solid foundation for our understanding of more realistic models.

Acknowledgments. We thank Junyi Chai for sharing the doubly periodic quasigeostrophic model code with us and Tsung-Lin Hsieh for the help of model setup on GFDL RDHPCS. We are grateful to Pablo ZuritaGotor for providing us the channel quasigeostrophic model code and his insightful discussions. Comments from Alistair Adcroft, Steve Garner, and two anonymous reviewers greatly improved the manuscript. CYC is supported by NSF Grant AGS-1733818.

\section{APPENDIX}

\section{Equations of the Model}

Following the notations of LH95, the potential vorticity $(Q)$ equations for the two equal-depth layers are

$$
\begin{aligned}
& \partial_{t} Q_{1}+J\left(\Psi_{1}, Q_{1}\right)=-\nu \nabla^{8} Q_{1}+\mathscr{D}_{1}, \\
& \partial_{t} Q_{2}+J\left(\Psi_{2}, Q_{2}\right)=-\nu \nabla^{8} Q_{2}+\mathscr{D}_{2},
\end{aligned}
$$

where the subscripts 1 and 2 refer to the top and bottom layers, respectively. The potential vorticity $Q$ are related to the total streamfunction $\Psi$ by

$$
\begin{aligned}
& Q_{1}=\nabla^{2} \Psi_{1}+k_{d}^{2}\left(\Psi_{2}-\Psi_{1}\right) / 2, \\
& Q_{2}=\nabla^{2} \Psi_{2}+k_{d}^{2}\left(\Psi_{1}-\Psi_{2}\right) / 2,
\end{aligned}
$$

and $\Psi$ contains a prescribed zonal mean part and an eddy part that varies in time and space:

$$
\begin{aligned}
& \Psi_{1}=-U y+\psi_{1}(x, y, t), \\
& \Psi_{2}=U y+\psi_{2}(x, y, t) .
\end{aligned}
$$

In the rhs of Eq. (A1), the subgrid-scale dissipation is parameterized as a $\nabla^{8}$ hyperviscosity and the largescale dissipation $\mathscr{D}$ is in a specific form of quadratic drag that assumes the surface stress is parallel to the eddy velocity (Grianik et al. 2004; Arbic and Scott 2008): 
$\mathscr{D}_{1}=-c_{D}\left[\partial_{x}\left(\left|\nabla \psi_{1}\right| \partial_{x} \psi_{1}\right)+\partial_{y}\left(\left|\nabla \psi_{1}\right| \partial_{y} \psi_{1}\right)\right]$

$\mathscr{D}_{2}=-c_{D}\left[\partial_{x}\left(\left|\nabla \psi_{2}\right| \partial_{x} \psi_{2}\right)+\partial_{y}\left(\left|\nabla \psi_{2}\right| \partial_{y} \psi_{2}\right)\right]$.

Altogether, the set of equations has four parameters: the wavenumber of deformation radius $k_{d}$, the mean vertical shear $U$, the hyperviscosity coefficient $\nu$, and the wavenumber of frictional length $c_{D}$. We choose to nondimensionalize the equations by $k_{d}$ and $U$ and leave the two dimensionless parameters as $\nu U^{-1} k_{d}^{7}$ and $c_{D} k_{d}^{-1}$. In this study, we have assumed the solutions (in the parameter space we explore) have no dependence on $\nu U^{-1} k_{d}^{7}$, since $\nu$ is set adaptively during the integration as described in Smith and Vallis (2002) and we do not have an explicit control on its value. This also assumes the solutions after nondimensionalization is independent of $U$, which is confirmed by Arbic and Scott (2008). The value of $U=0.005$ that we use is simply chosen for convenience in the comparison with the LH95 simulations.

\section{REFERENCES}

Arbic, B. K., and G. R. Flierl, 2004: Baroclinically unstable geostrophic turbulence in the limits of strong and weak bottom Ekman friction: Application to midocean eddies. J. Phys. Oceanogr., 34, 2257-2273, https://doi.org/10.1175/ 1520-0485(2004)034<2257:BUGTIT>2.0.CO;2.

_- and R. B. Scott, 2008: On quadratic bottom drag, geostrophic turbulence, and oceanic mesoscale eddies. J. Phys. Oceanogr., 38, 84-103, https://doi.org/10.1175/2007JPO3653.1.

Barry, L., G. C. Craig, and J. Thuburn, 2002: Poleward heat transport by the atmospheric heat engine. Nature, 415, 774777, https://doi.org/10.1038/415774a.

Chai, J., 2016: Understanding geostrophic turbulence in a hierarchy of models. Ph.D. thesis, Princeton University, 211 pp.

Grianik, N., I. M. Held, K. S. Smith, and G. K. Vallis, 2004: The effects of quadratic drag on the inverse cascade of twodimensional turbulence. Phys. Fluids, 16, 73-78, https:// doi.org/10.1063/1.1630054.

Haidvogel, D. B., and I. M. Held, 1980: Homogeneous quasigeostrophic turbulence driven by a uniform temperature gradient. J. Atmos. Sci., 37, 2644-2660, https://doi.org/10.1175/ 1520-0469(1980)037<2644:HQGTDB>2.0.CO;2.

Held, I. M., 1999: The macroturbulence of the troposphere. Tellus, 51, 59-70, https://doi.org/10.3402/tellusa.v51i1.12306.

__ , and V. D. Larichev, 1996: A scaling theory for horizontally homogeneous, baroclinically unstable flow on a beta plane. J. Atmos. Sci., 53, 946-952, https://doi.org/10.1175/ 1520-0469(1996)053<0946:ASTFHH>2.0.CO;2.
Jansen, M., and R. Ferrari, 2013: Equilibration of an atmosphere by adiabatic eddy fluxes. J. Atmos. Sci., 70, 2948-2962, https:// doi.org/10.1175/JAS-D-13-013.1.

— A. A. Adcroft, R. Hallberg, and I. M. Held, 2015: Parameterization of eddy fluxes based on a mesoscale energy budget. Ocean Modell., 92, 28-41, https://doi.org/10.1016/ j.ocemod.2015.05.007.

Lapeyre, G., and I. M. Held, 2003: Diffusivity, kinetic energy dissipation, and closure theories for the poleward eddy heat flux. J. Atmos. Sci., 60, 2907-2916, https://doi.org/10.1175/15200469(2003)060<2907:DKEDAC > 2.0.CO;2.

Larichev, V. D., and I. M. Held, 1995: Eddy amplitudes and fluxes in a homogeneous model of fully developed baroclinic instability. J. Phys. Oceanogr., 25, 2285-2297, https://doi.org/ 10.1175/1520-0485(1995)025<2285:EAAFIA >2.0.CO;2.

Pavan, V., and I. M. Held, 1996: The diffusive approximation for eddy fluxes in baroclinically unstable jets. J. Atmos. Sci., 53, 1262-1272, https://doi.org/10.1175/1520-0469(1996)053<1262: TDAFEF $>2.0 . \mathrm{CO} ; 2$.

Phillips, N. A., 1956: The general circulation of the atmosphere: A numerical experiment. Quart. J. Roy. Meteor. Soc., 82, 123-164, https://doi.org/10.1002/qj.49708235202.

Rhines, P. B., 1975: Waves and turbulence on a beta-plane. J. Fluid Mech., 69, 417-443, https://doi.org/10.1017/ S0022112075001504.

_ 1977: The dynamics of unsteady currents. Marine Modeling, E. D. Goldberg et al., Eds., The Sea-Ideas and Observations on Progress in the Study of the Seas, Vol. 6, John Wiley and Sons, 189-318.

Salmon, R., 1978: Two-layer quasi-geostrophic turbulence in a simple special case. Geophys. Astrophys. Fluid Dyn., 10, 2552, https://doi.org/10.1080/03091927808242628.

_ 1980: Baroclinic instability and geostrophic turbulence. Geophys. Astrophys. Fluid Dyn., 15, 167-211, https://doi.org/ 10.1080/03091928008241178.

Smith, K. S., and G. K. Vallis, 2002: The scales and equilibration of midocean eddies: Forced-dissipative flow. J. Phys. Oceanogr., 32, 1699-1720, https://doi.org/10.1175/15200485(2002)032<1699:TSAEOM > 2.0.CO;2.

Stone, P. H., 1972: A simplified radiative-dynamical model for the static stability of rotating atmospheres. J. Atmos. Sci., 29, 405-418, https:// doi.org/10.1175/1520-0469(1972)029<0405:ASRDMF>2.0.CO;2.

Thompson, A. F., and W. R. Young, 2006: Scaling baroclinic eddy fluxes: Vortices and energy balance. J. Phys. Oceanogr., 36, 720-738, https://doi.org/10.1175/JPO2874.1.

— , and — 2007: Two-layer baroclinic eddy heat fluxes: Zonal flows and energy balance. J. Atmos. Sci., 64, 3214-3231, https:// doi.org/10.1175/JAS4000.1.

Zurita-Gotor, P., 2007: The relation between baroclinic adjustment and turbulent diffusion in the two-layer model. J. Atmos. Sci., 64, 1284 1300, https://doi.org/10.1175/JAS3886.1.

, J. Blanco-Fuentes, and E. P. Gerber, 2014: The impact of baroclinic eddy feedback on the persistence of jet variability in the two-layer model. J. Atmos. Sci., 71, 410-429, https:// doi.org/10.1175/JAS-D-13-0102.1. 